\title{
Dicyano-Imidazole-Based Host Materials Possessing Balanced Bipolar Nature to Realize Efficient OLEDs with Extremely High Luminance
}

Rong-Huei Yi, ${ }^{\mathrm{a}+}$ Chang-Min Shao, ${ }^{\mathrm{b}+}$ Chien-Hsiang Lin, ${ }^{\mathrm{a}}$ Yu-Chuan Fang, ${ }^{\mathrm{a}}$ HsiangLing Shen, ${ }^{a}$ Chin-Wei Lu, ${ }^{* a}$ Kang-Yu Wang, ${ }^{b}$ Chih-Hao Chang, ${ }^{* b}$ Li-Yin Chen ${ }^{* c}$, and Yong-Hsiang Chang ${ }^{\mathrm{d}}$

${ }^{a}$ Department of Applied Chemistry, Providence University, Taichung 43301, Taiwan

${ }^{b}$ Department of Electrical Engineering, Yuan Ze University, Chungli 32003, Taiwan

${ }^{c}$ Department of Photonics, National Chiao Tung University, Hsinchu 30010, Taiwan

${ }^{\mathrm{d}}$ Department of Photonics, National Sun Yat-sen University, Kaohsiung 80424, Taiwan

${ }^{+}$Equal contribution

To whom to correspond:

E-mail: cwlu@pu.edu.tw; Tel: 886-4-26328001-15213

E-mail: chc@saturn.yzu.edu.tw; Tel: 886-3-4638800-7501

E-mail: 1y_chen@mail.nsysu.edu.tw; Tel: 886-7-5252000-4462

\section{Contents}

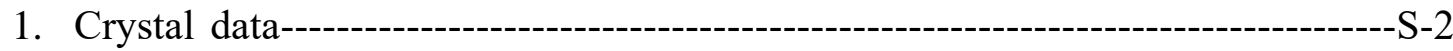

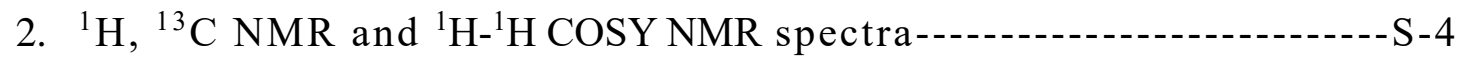

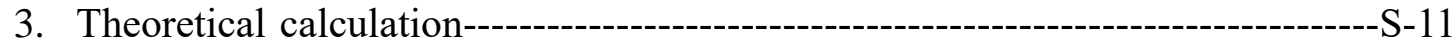

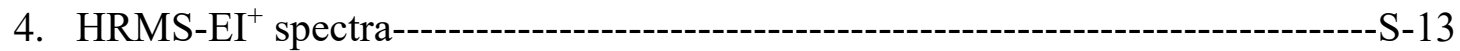

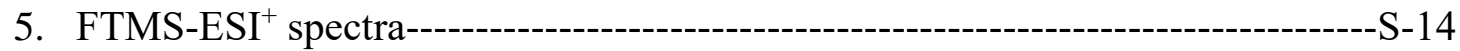

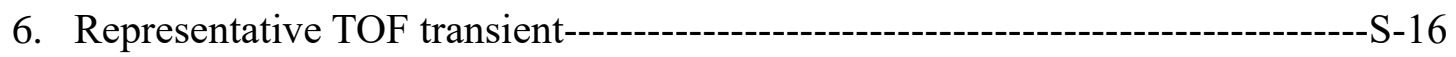


Table S1. Crystal data of compounds imM-o-Cz, imM-m-Cz, and imM-p-Cz.

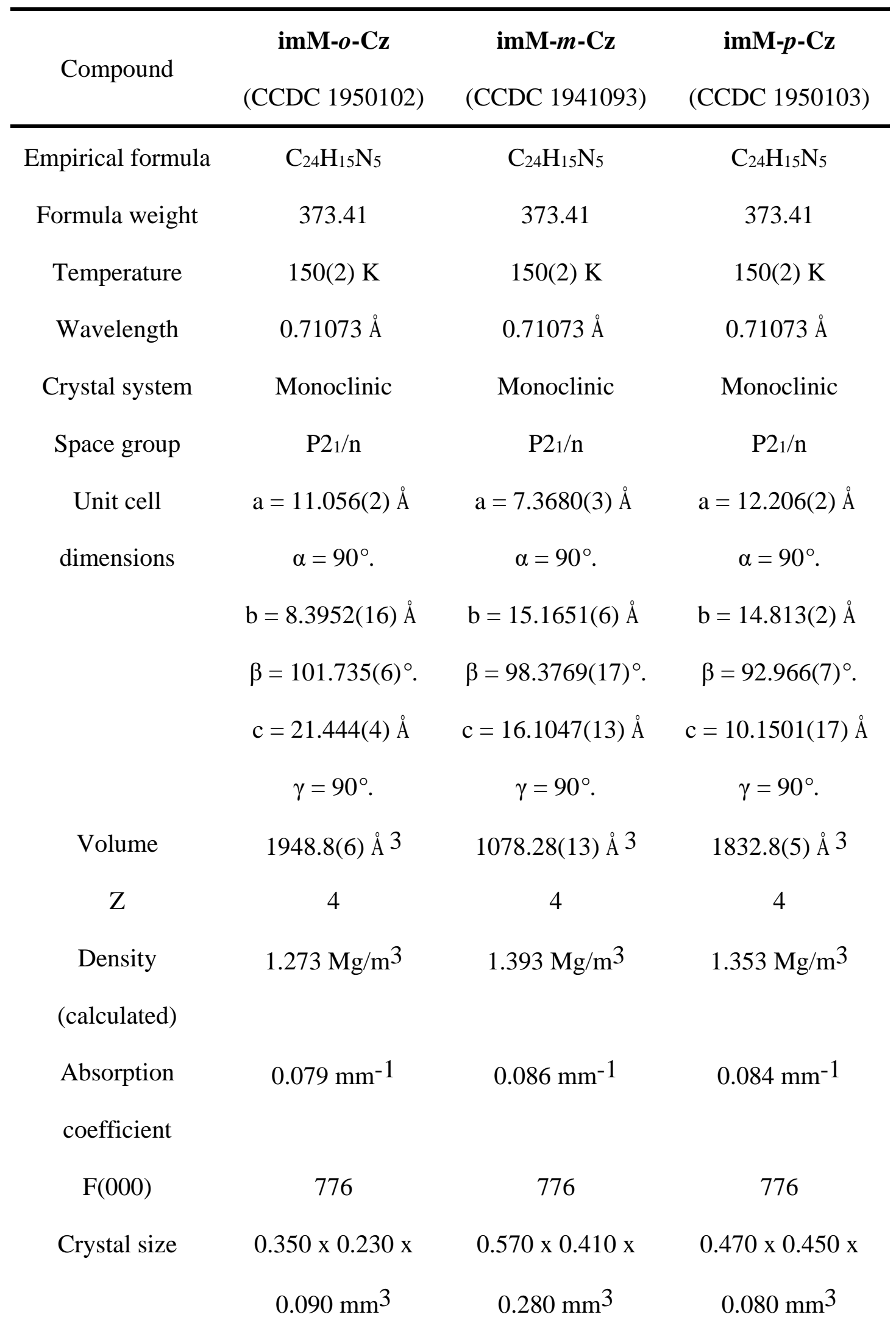

Theta range for $\quad 3.103$ to $27.456^{\circ}$. $\quad 2.957$ to $27.118^{\circ}$. 3.012 to $27.951^{\circ}$. 


\begin{tabular}{|c|c|c|c|}
\hline \multirow[t]{3}{*}{ Index ranges } & $-14<=\mathrm{h}<=14,-$ & $-9<=\mathrm{h}<=9,-$ & $-16<=\mathrm{h}<=16,-$ \\
\hline & $10<=\mathrm{k}<=9,-$ & $19<=\mathrm{k}<=19,-$ & $19<=\mathrm{k}<=19,-$ \\
\hline & $27<=1<=27$ & $20<=1<=20$ & $13<=1<=13$ \\
\hline Reflections & 29594 & 31369 & 30722 \\
\hline collected & & & \\
\hline Independent & $4398[\mathrm{R}($ int $)=$ & $3915[\mathrm{R}$ (int) $=$ & $4380[\mathrm{R}($ int $)=$ \\
\hline reflections & $0.0925]$ & $0.0429]$ & $0.0356]$ \\
\hline Completeness to & $99.6 \%$ & $99.4 \%$ & $99.4 \%$ \\
\hline \multicolumn{4}{|l|}{ theta $=25.242^{\circ}$} \\
\hline Absorption & Semi-empirical & Semi-empirical & Semi-empirical \\
\hline correction & from equivalents & from equivalents & from equivalents \\
\hline Max. and min. & 0.9281 and 0.7692 & 0.9281 and 0.8501 & 0.9281 and 0.8275 \\
\hline \multicolumn{4}{|l|}{ transmission } \\
\hline Refinement & Full-matrix least- & Full-matrix least- & Full-matrix least- \\
\hline method & squares on $\mathrm{F}^{2}$ & squares on $\mathrm{F}^{2}$ & squares on $\mathrm{F}^{2}$ \\
\hline Data / restraints / & 4398 / 0 / 263 & 3915 / 0 / 262 & 4380 / 0 / 262 \\
\hline \multicolumn{4}{|l|}{ parameters } \\
\hline Goodness-of-fit & 1.070 & 0.641 & 1.014 \\
\hline \multicolumn{4}{|l|}{ on $\mathrm{F}^{2}$} \\
\hline Final $\mathrm{R}$ indices & $\mathrm{R} 1=0.1024, \mathrm{wR} 2$ & $\mathrm{R} 1=0.0445, \mathrm{wR} 2$ & $\mathrm{R} 1=0.0468, \mathrm{wR} 2$ \\
\hline$[\mathrm{I}>2 \operatorname{sigma}(\mathrm{I})]$ & $=0.2791$ & $=0.1330$ & $=0.1338$ \\
\hline $\mathrm{R}$ indices (all & $\mathrm{R} 1=0.1561, \mathrm{wR} 2$ & $\mathrm{R} 1=0.0530, \mathrm{wR} 2$ & $\mathrm{R} 1=0.0651, \mathrm{wR} 2$ \\
\hline data) & $=0.3212$ & $=0.1525$ & $=0.1558$ \\
\hline Extinction & $0.043(7)$ & $\mathrm{n} / \mathrm{a}$ & $\mathrm{n} / \mathrm{a}$ \\
\hline
\end{tabular}


coefficient

Largest diff. peak $\quad 0.474$ and $-0.484 \quad 0.480$ and $-0.365 \quad 0.475$ and -0.381 and hole

e. $\AA^{-3}$

e. $\AA^{-3}$

e. $\AA^{-3}$

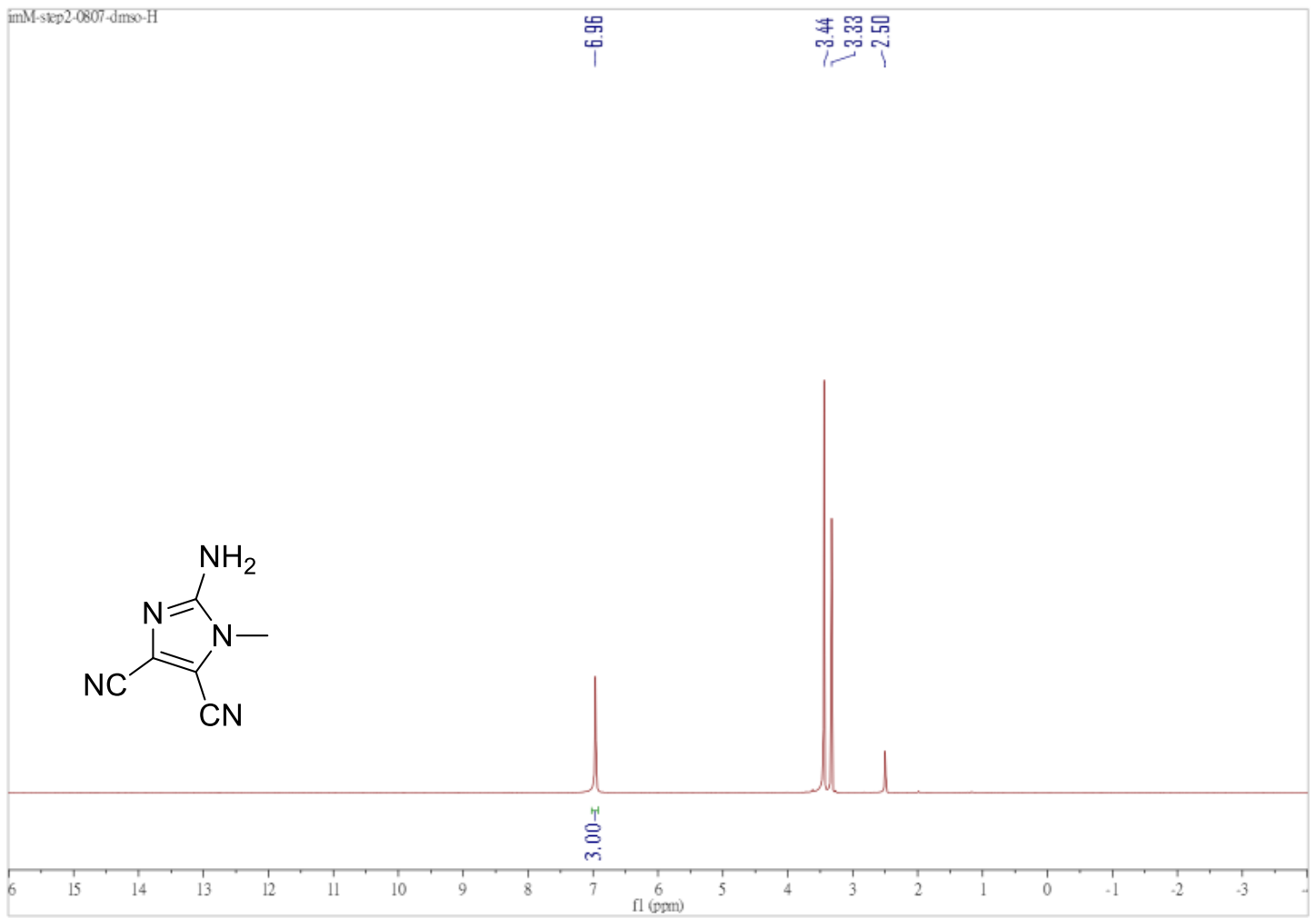

Figure S1. ${ }^{1} \mathrm{H}$ NMR spectrum for compound 1 (400 MHz, DMSO- $d_{6}, 298 \mathrm{~K}$ ). 


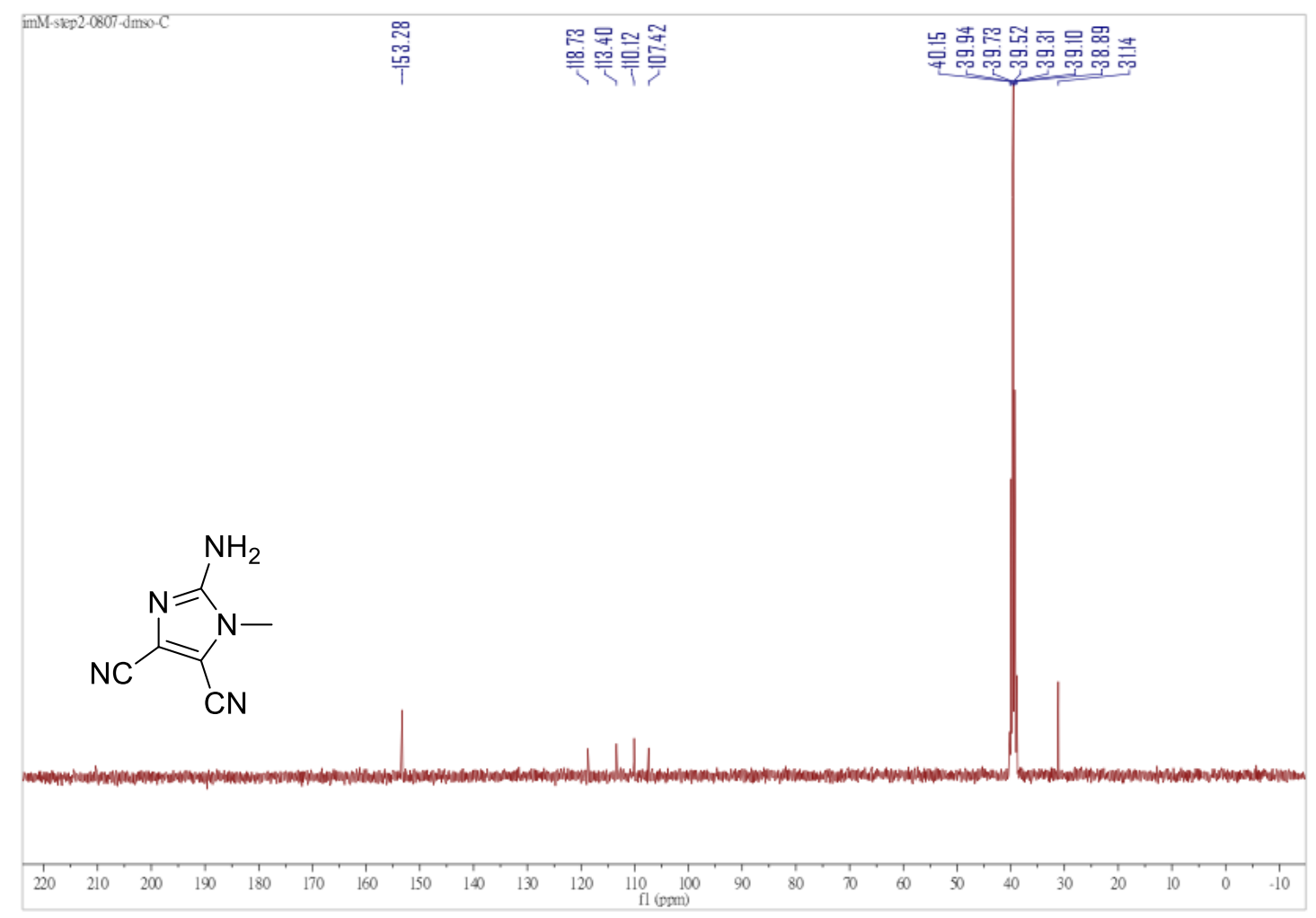

Figure S2. ${ }^{13} \mathrm{C}$ NMR spectrum for compound 1 (100 MHz, DMSO-d6, $298 \mathrm{~K}$ ).

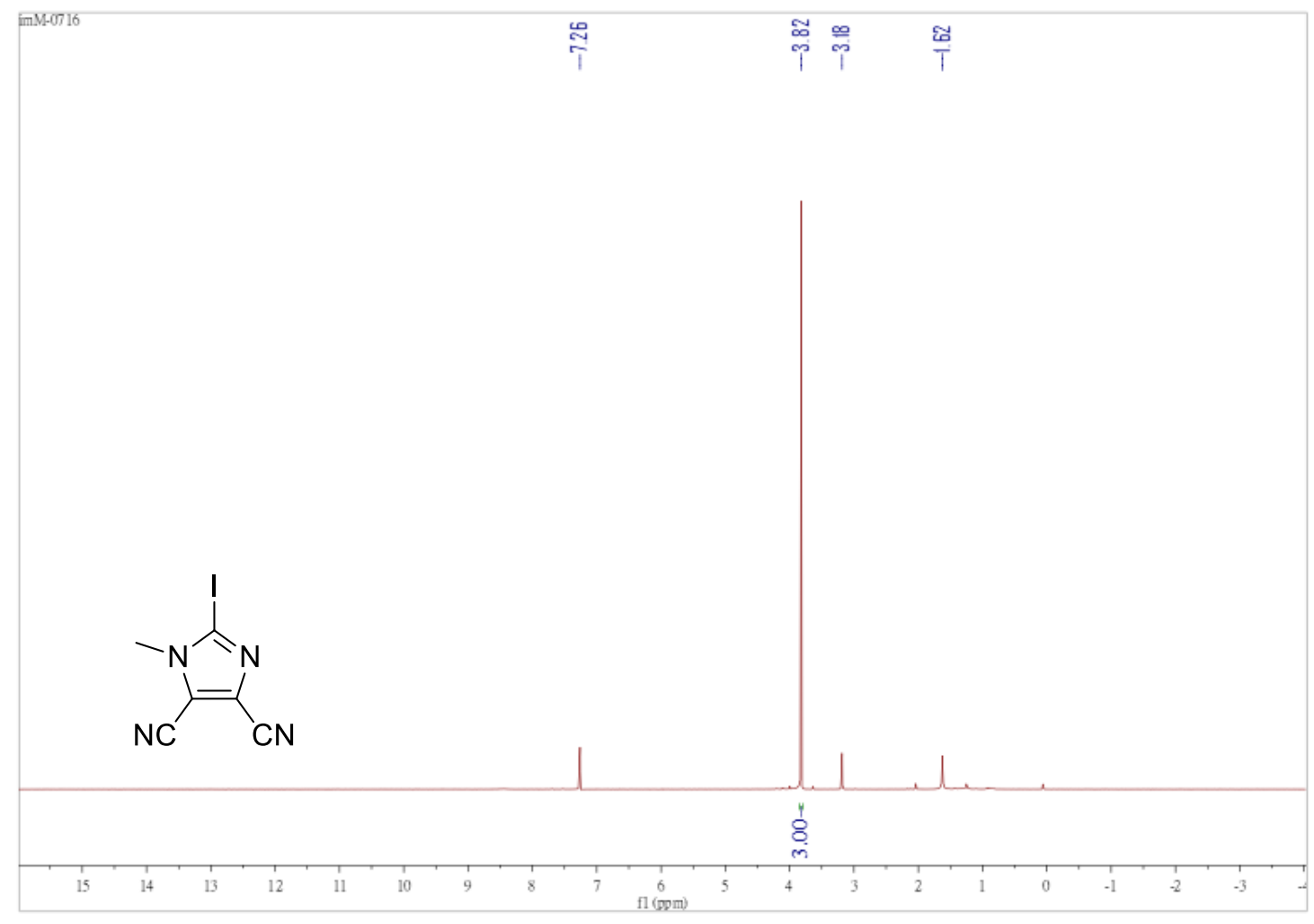

Figure S3. ${ }^{1} \mathrm{H}$ NMR spectrum for compound 2 (400 MHz, $\mathrm{CDCl}_{3}, 298 \mathrm{~K}$ ). 


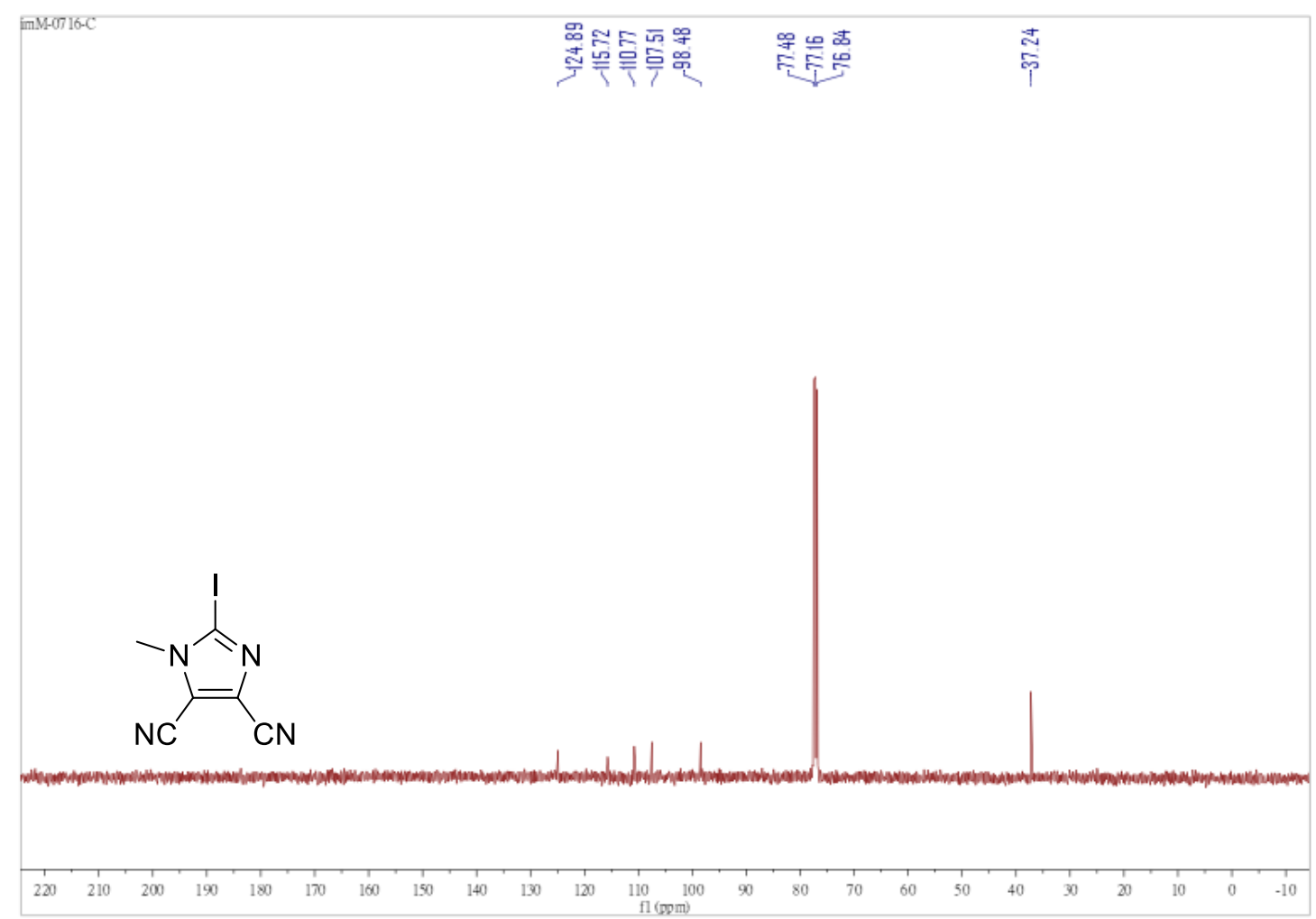

Figure S4. ${ }^{13} \mathrm{C}$ NMR spectrum for compound $2\left(100 \mathrm{MHz}, \mathrm{CDCl}_{3}, 298 \mathrm{~K}\right)$.

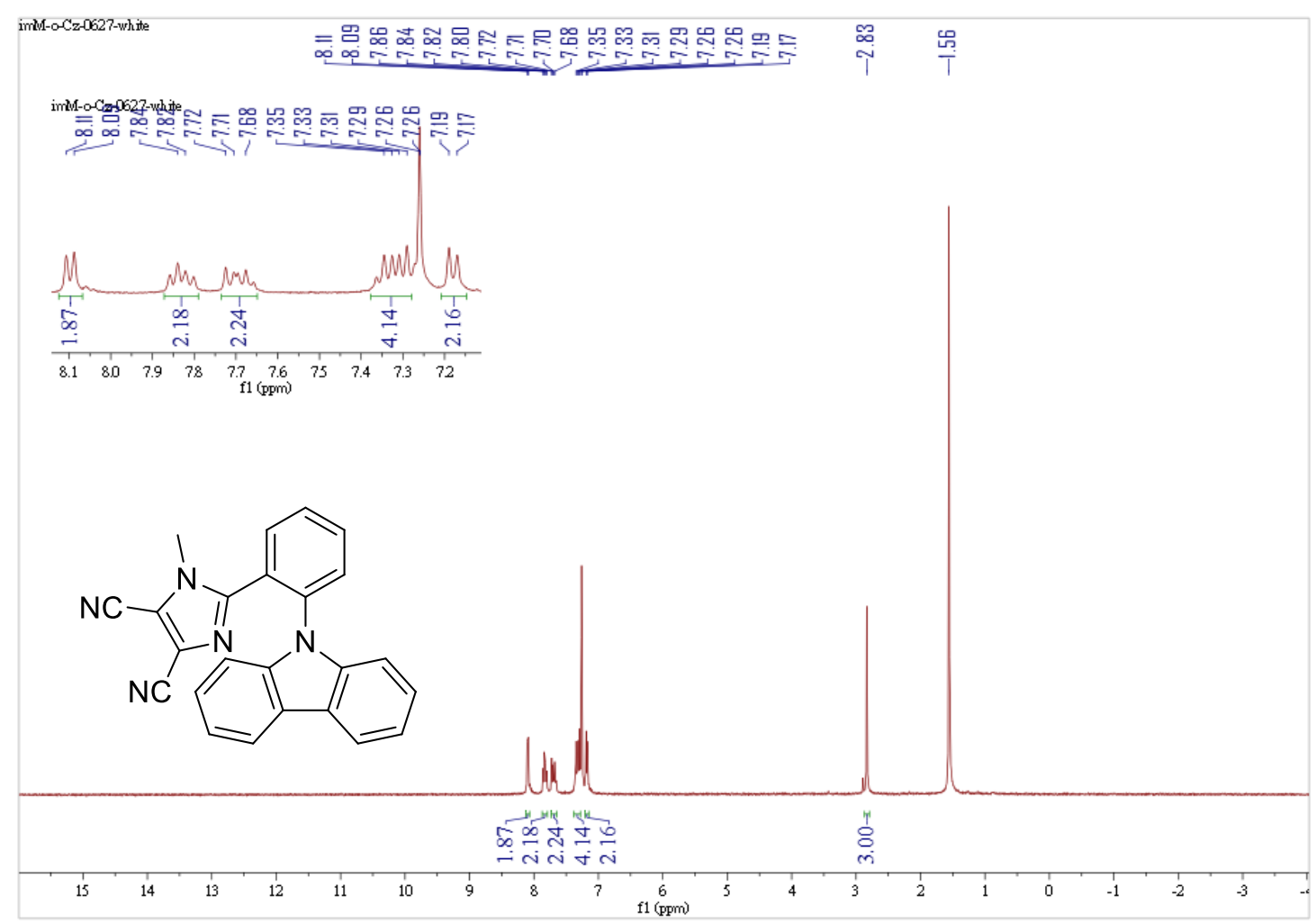

Figure S5. ${ }^{1} \mathrm{H}$ NMR spectrum for imM-o-Cz (400 MHz, $\left.\mathrm{CDCl}_{3}, 298 \mathrm{~K}\right)$. 


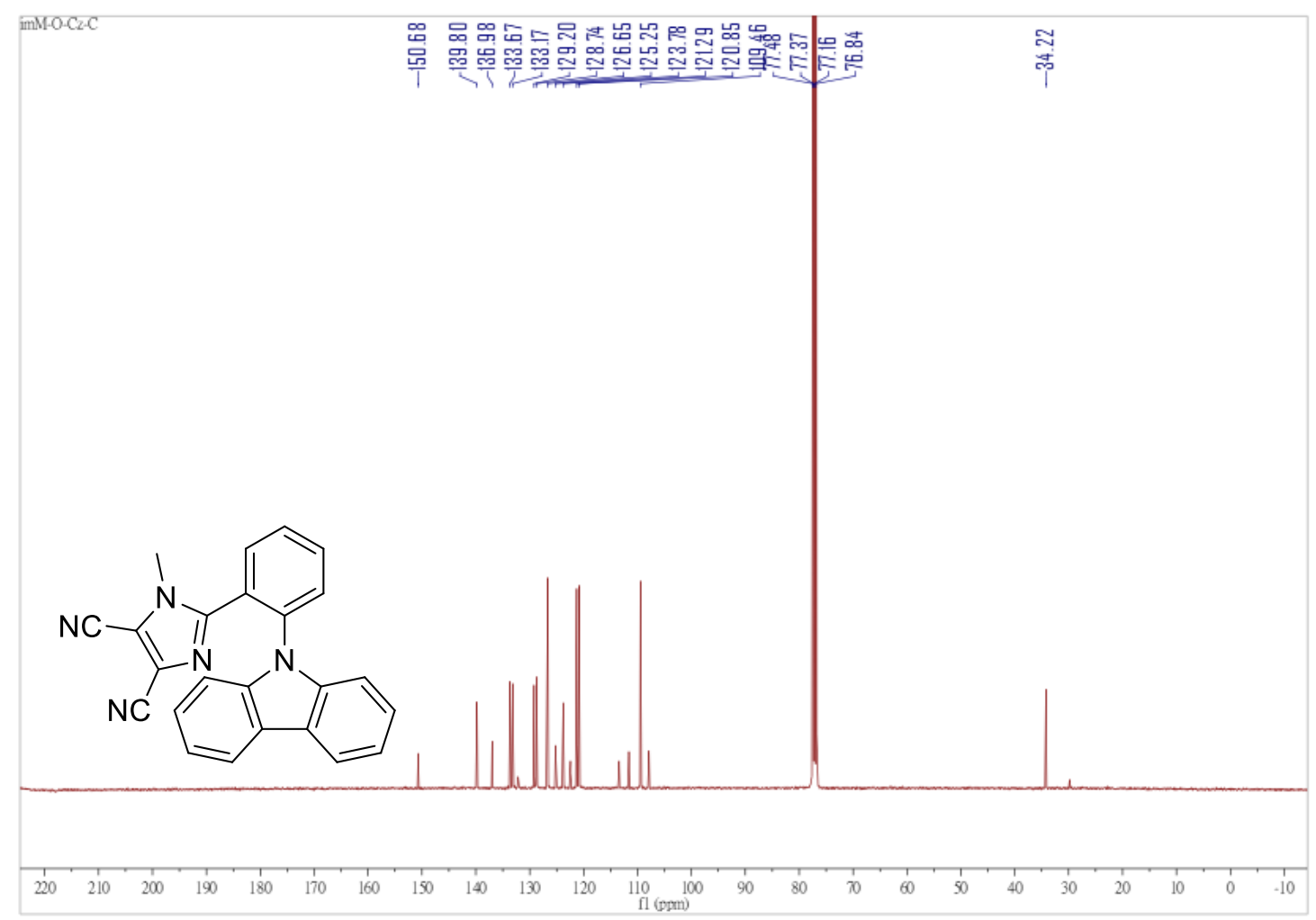

Figure S6. ${ }^{13} \mathrm{C}$ NMR spectrum for imM-o-Cz $\left(100 \mathrm{MHz}, \mathrm{CDCl}_{3}, 298 \mathrm{~K}\right)$.

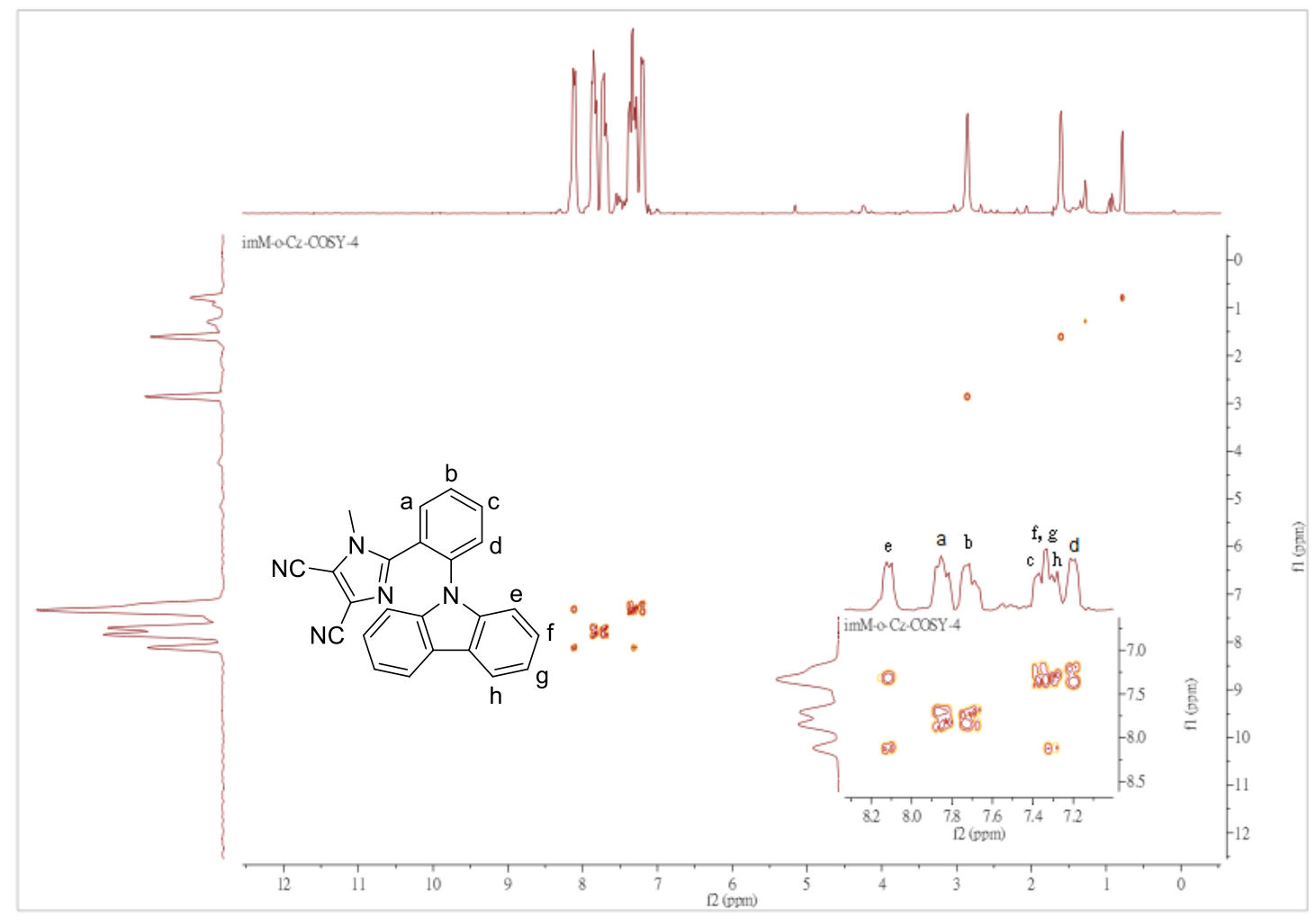

Figure S7. ${ }^{1} \mathrm{H}-{ }^{1} \mathrm{H}$ COSY spectrum $(400 \mathrm{MHz}$, pulse width of $14.0 \mu \mathrm{s})$ of imM-o-Cz in $\mathrm{CDCl}_{3}$ at $298 \mathrm{~K}$, the inset is the expansion of aromatic region. 


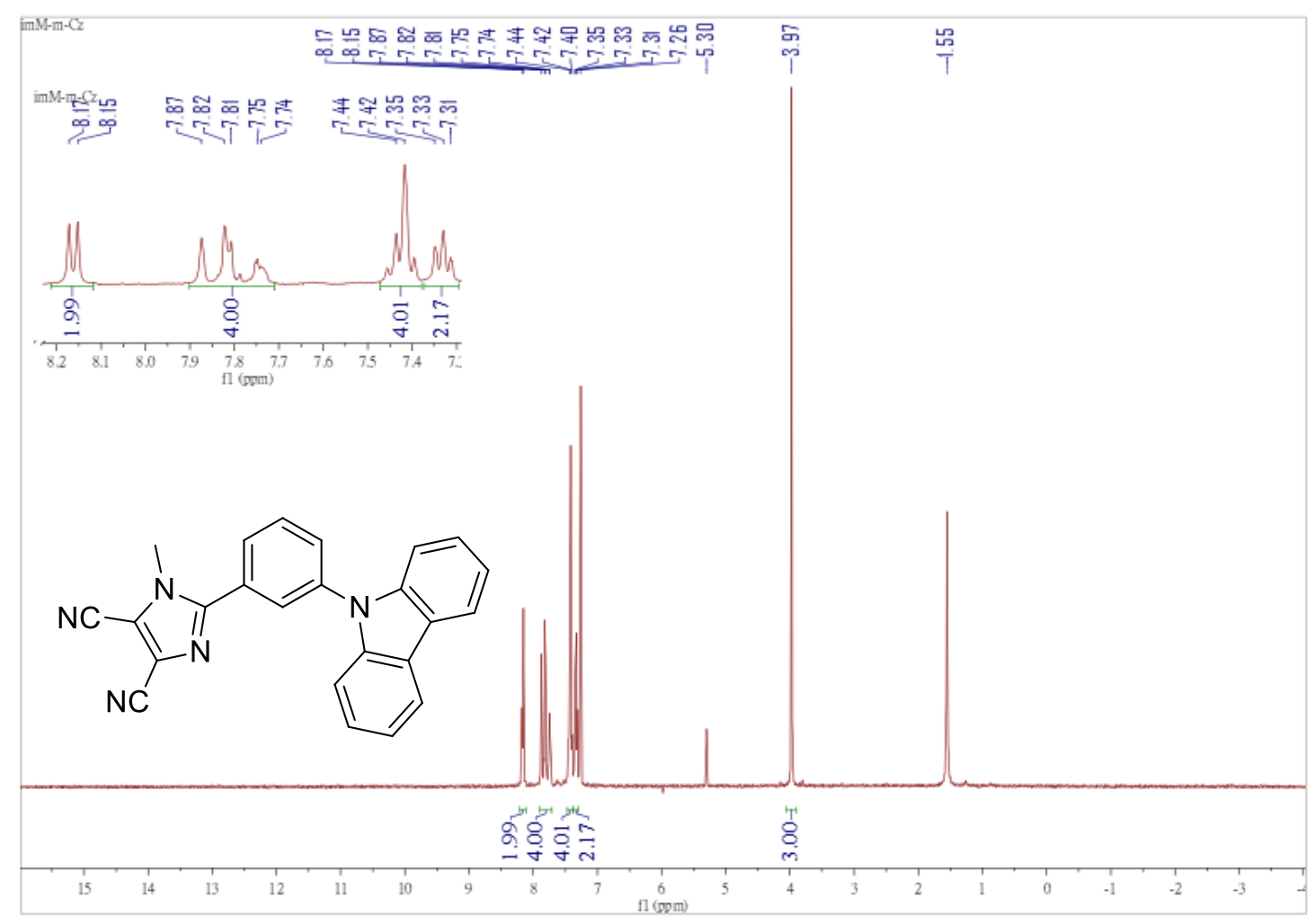

Figure S8. ${ }^{1} \mathrm{H}$ NMR spectrum for $\mathbf{i m M}-\boldsymbol{m}-\mathbf{C z}\left(400 \mathrm{MHz}, \mathrm{CDCl}_{3}, 298 \mathrm{~K}\right)$.

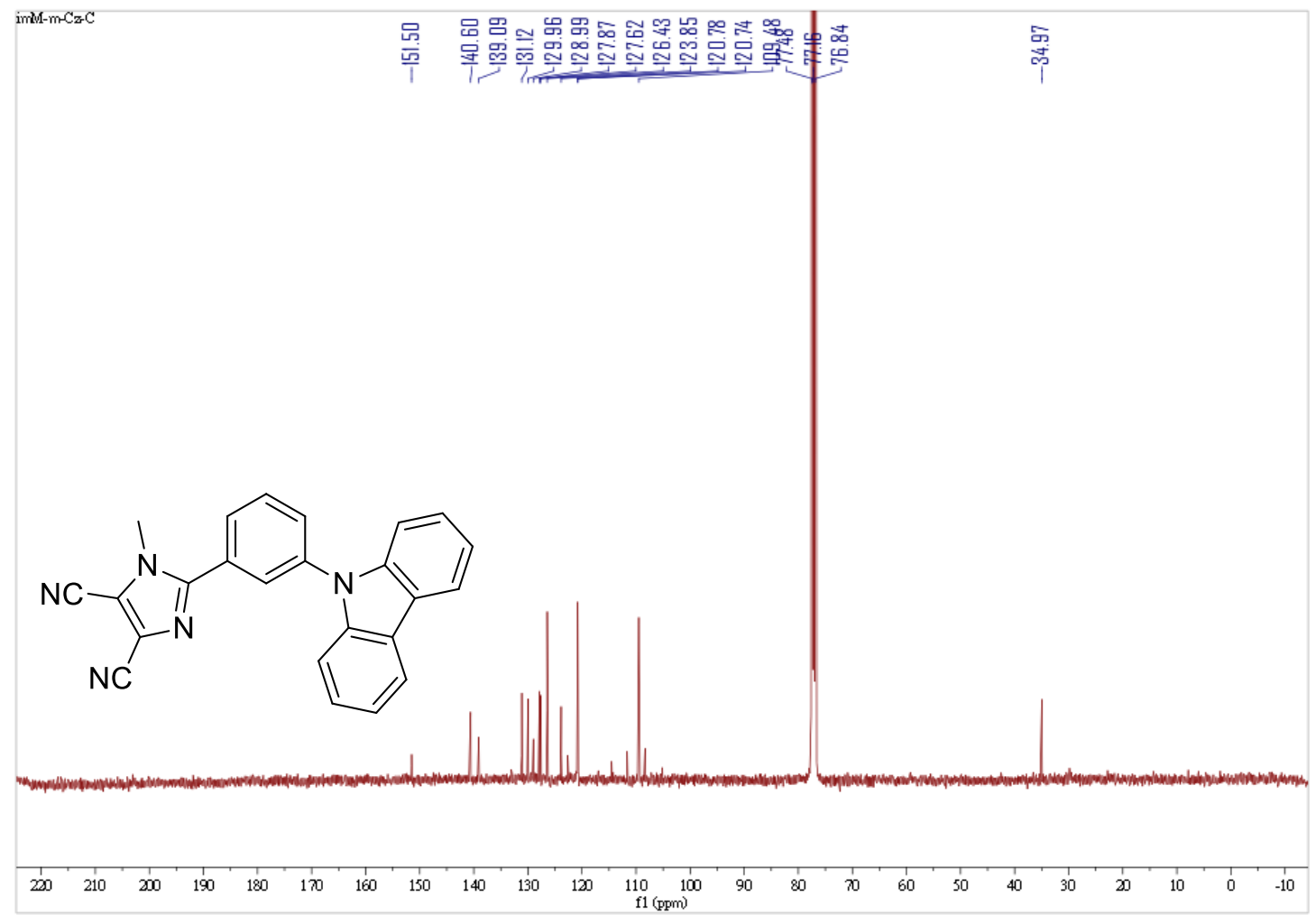

Figure S9. ${ }^{13} \mathrm{C}$ NMR spectrum for imM-m-Cz (100 MHz, $\left.\mathrm{CDCl}_{3}, 298 \mathrm{~K}\right)$. 


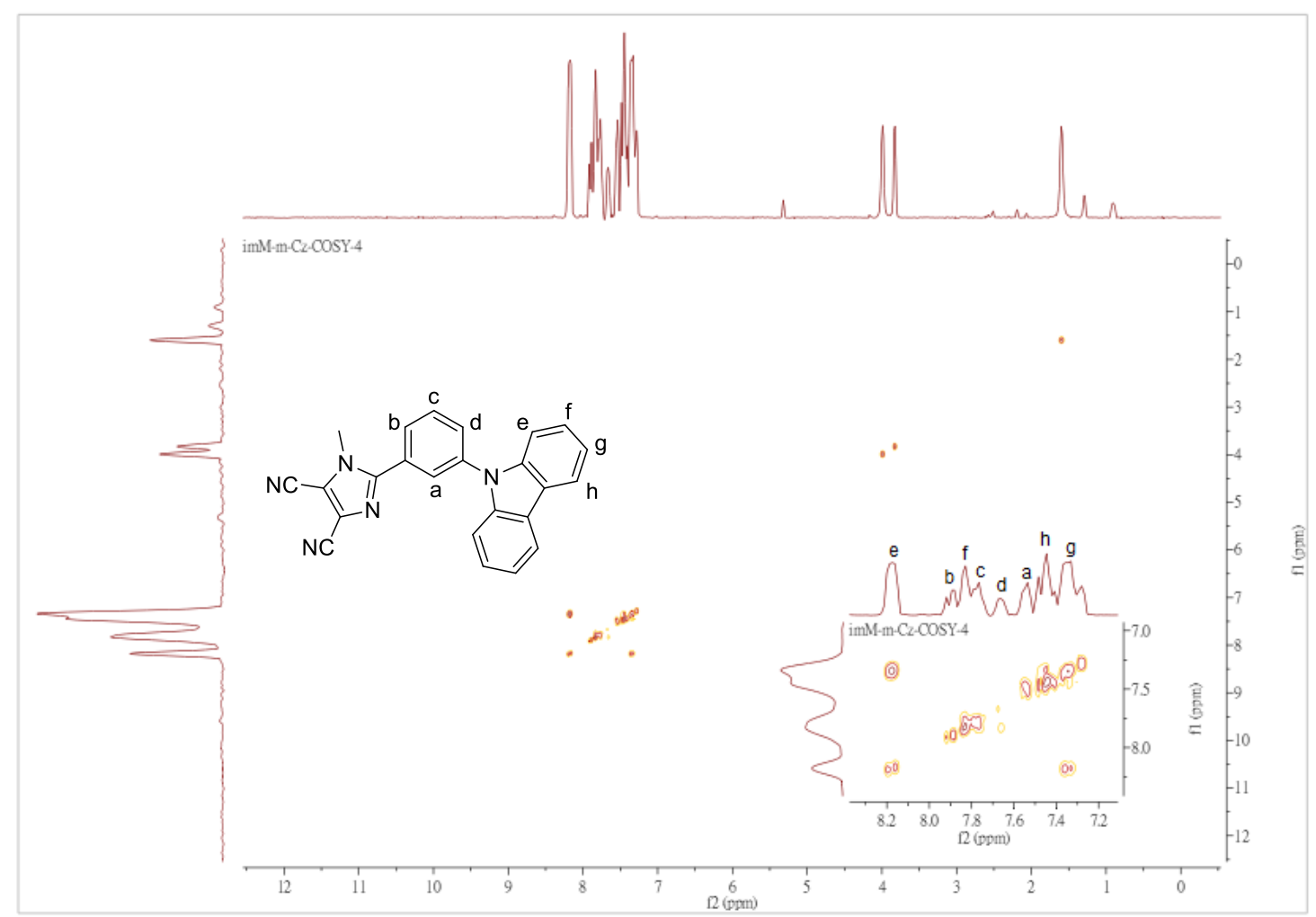

Figure S10. ${ }^{1} \mathrm{H}-{ }^{1} \mathrm{H}$ COSY spectrum $(400 \mathrm{MHz}$, pulse width of $14.0 \mu \mathrm{s})$ of imM-m-Cz in $\mathrm{CDCl}_{3}$ at $298 \mathrm{~K}$, the inset is the expansion of aromatic region.

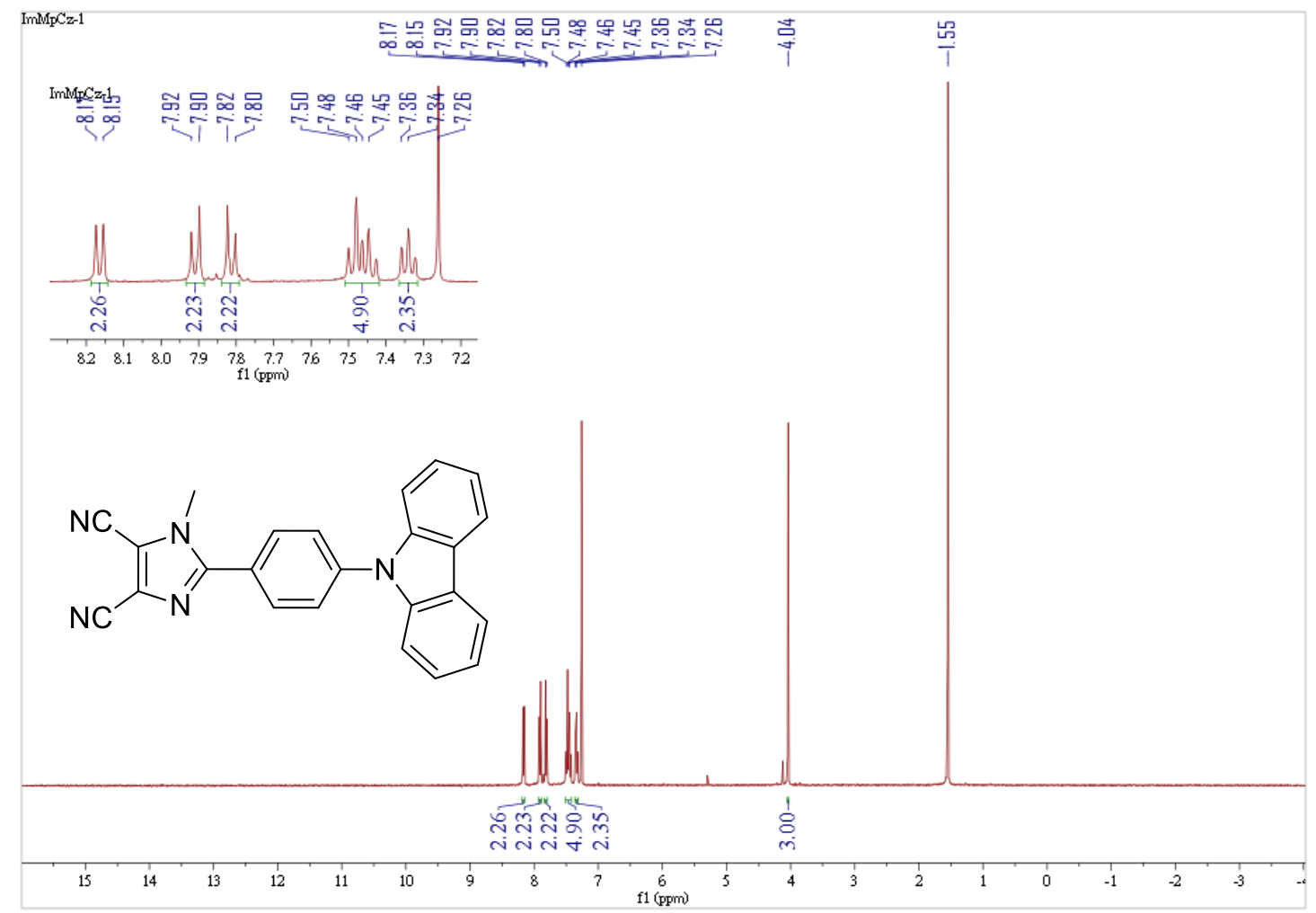

Figure S11. ${ }^{1} \mathrm{H}$ NMR spectrum for imM-p-Cz (400 MHz, $\left.\mathrm{CDCl}_{3}, 298 \mathrm{~K}\right)$. 


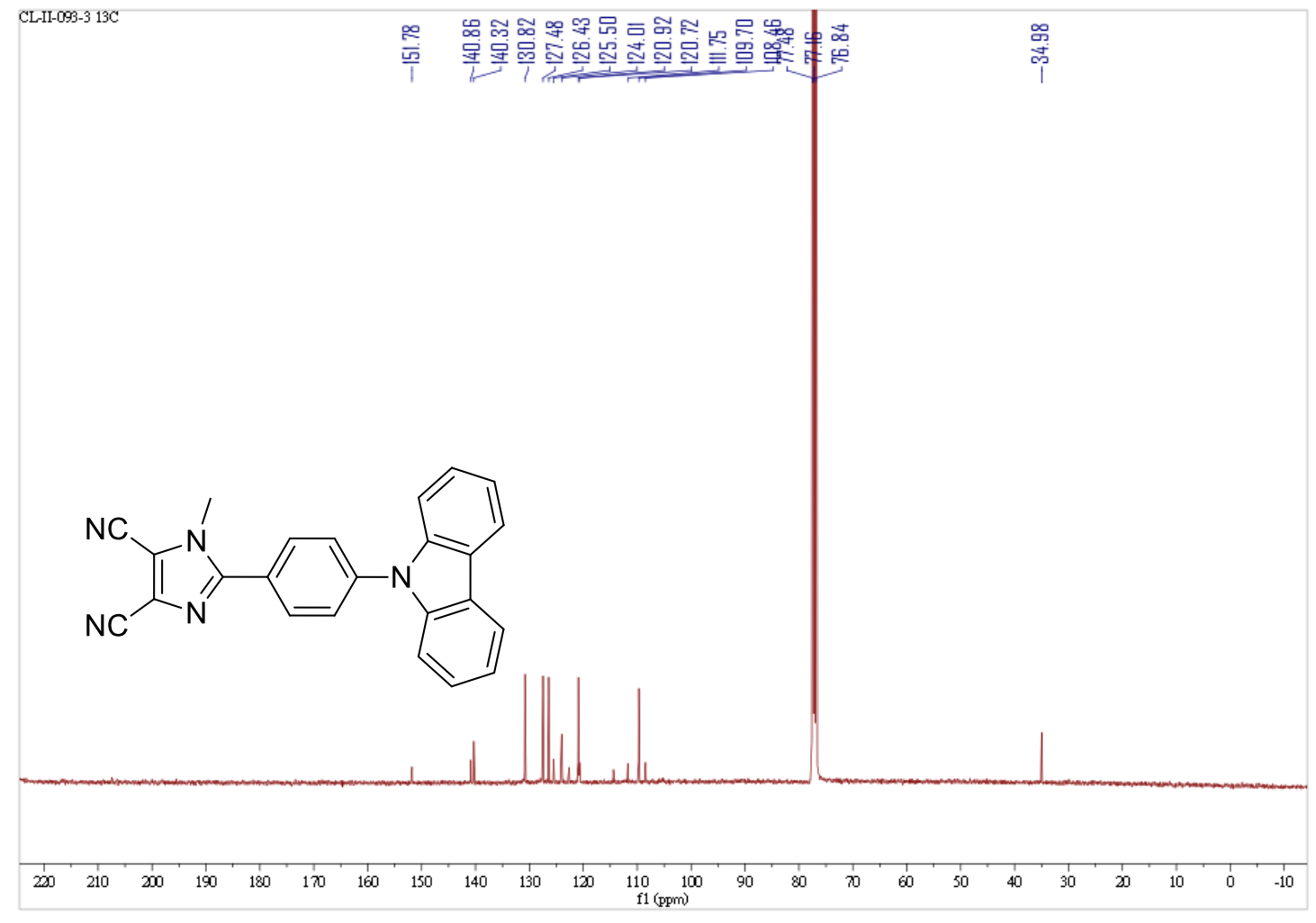

Figure S12. ${ }^{13} \mathrm{C}$ NMR spectrum for imM-p-Cz (100 MHz, $\left.\mathrm{CDCl}_{3}, 298 \mathrm{~K}\right)$.

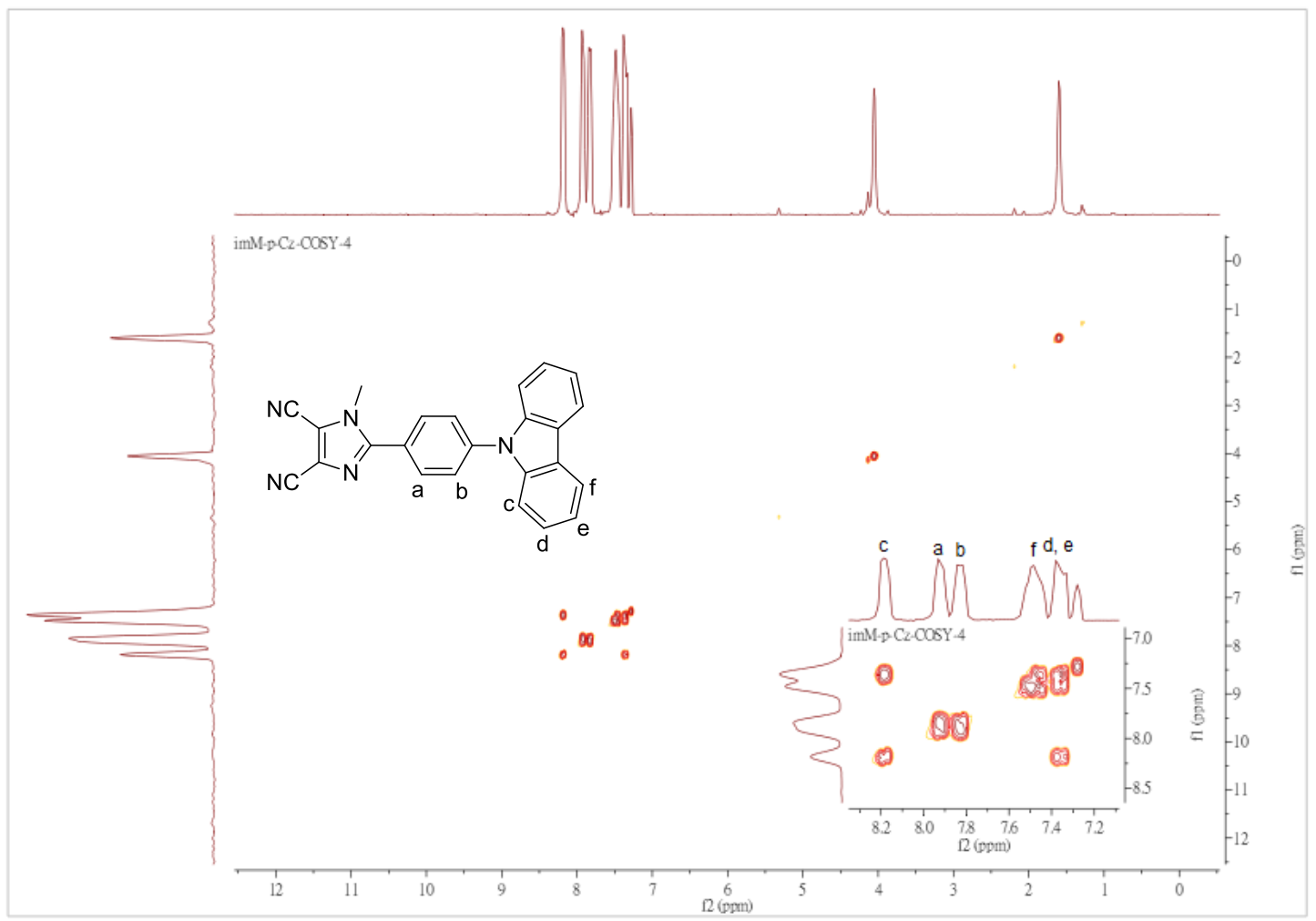


Figure S13. ${ }^{1} \mathrm{H}-{ }^{1} \mathrm{H}$ COSY spectrum $(400 \mathrm{MHz}$, pulse width of $14.0 \mu \mathrm{s})$ of imM-p-Cz in $\mathrm{CDCl}_{3}$ at $298 \mathrm{~K}$, the inset is the expansion of aromatic region.

Table S2. Calculated singlet and triplet states for imM-o-Cz, imM-m-Cz, and imM-pCz by TDDFT approach.

\begin{tabular}{|c|c|c|c|c|c|}
\hline Compound & State & $\begin{array}{c}\text { Dihedral } \\
\text { angle }\end{array}$ & $\begin{array}{c}\mathrm{eV} \\
\left(\lambda_{\mathrm{cal}}, \mathrm{nm}\right)^{\mathrm{a}}\end{array}$ & $f^{b}$ & $\begin{array}{l}\text { Ground state } \\
\text { dipole moment } \\
\text { (D) }\end{array}$ \\
\hline \multirow{3}{*}{$\operatorname{imM-o-Cz}$} & $\mathrm{S}_{1}$ & & $3.21(387)$ & 0.0001 & \multirow{3}{*}{10.7828} \\
\hline & & 108.0 & & & \\
\hline & $\mathrm{T}_{1}$ & & $2.53(491)$ & 0.0000 & \\
\hline \multirow{3}{*}{ imM-m-Cz } & $\mathrm{S}_{1}$ & & $2.63(472)$ & 0.0056 & \multirow{3}{*}{11.6431} \\
\hline & & 115.2 & & & \\
\hline & $\mathrm{T}_{1}$ & & 2.07 (599) & 0.0000 & \\
\hline \multirow{3}{*}{$\operatorname{imM-p-Cz}$} & $\mathrm{S}_{1}$ & & $2.71(457)$ & 0.6281 & \multirow{3}{*}{15.6676} \\
\hline & & 115.2 & & & \\
\hline & $\mathrm{T}_{1}$ & & $2.01(618)$ & 0.0000 & \\
\hline
\end{tabular}

${ }^{a}$ Calculated excitation energies for the singlet and triplet states in DCM solution. ${ }^{b}$ Simulated singlet and triplet oscillator strength.
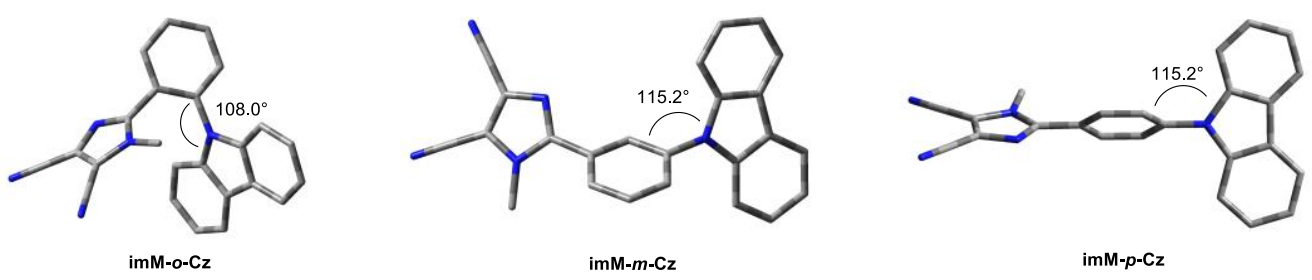

Figure S14. Ground state geometries of compounds imM-o-Cz, imM-m-Cz, and imM- 
p-Cz obtained at B3LYB/6-31G(d,p) level of theory. Hydrogen atoms are omitted for clarity. 
Data : 14013 Date : 19-Aug-2019 15:54
Instrument: MStation

Note :
llet : Direct lon Mode : El

Spectrum Type : Normal lon [EF-Linear]
RT : 0.06 min Scant : (2.4) Temp : 3276.7 deg. C

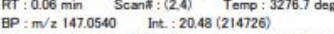

Output $\mathrm{m} / \mathrm{z}$ range $: 125$ to 165 Cut Level $: 0.00$ s

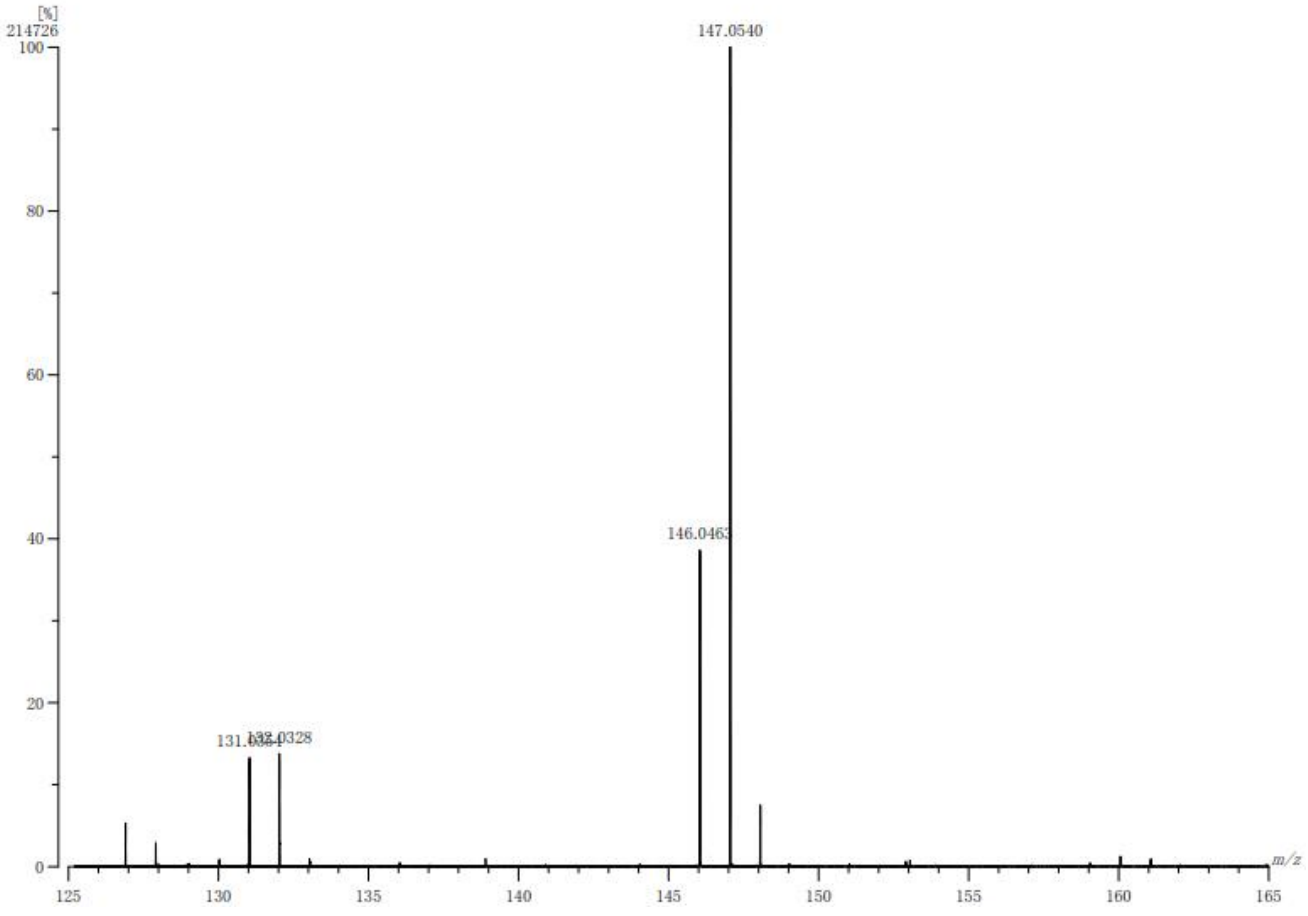

Figure S15. HRMS-EI ${ }^{+}$spectrum of compound 1.

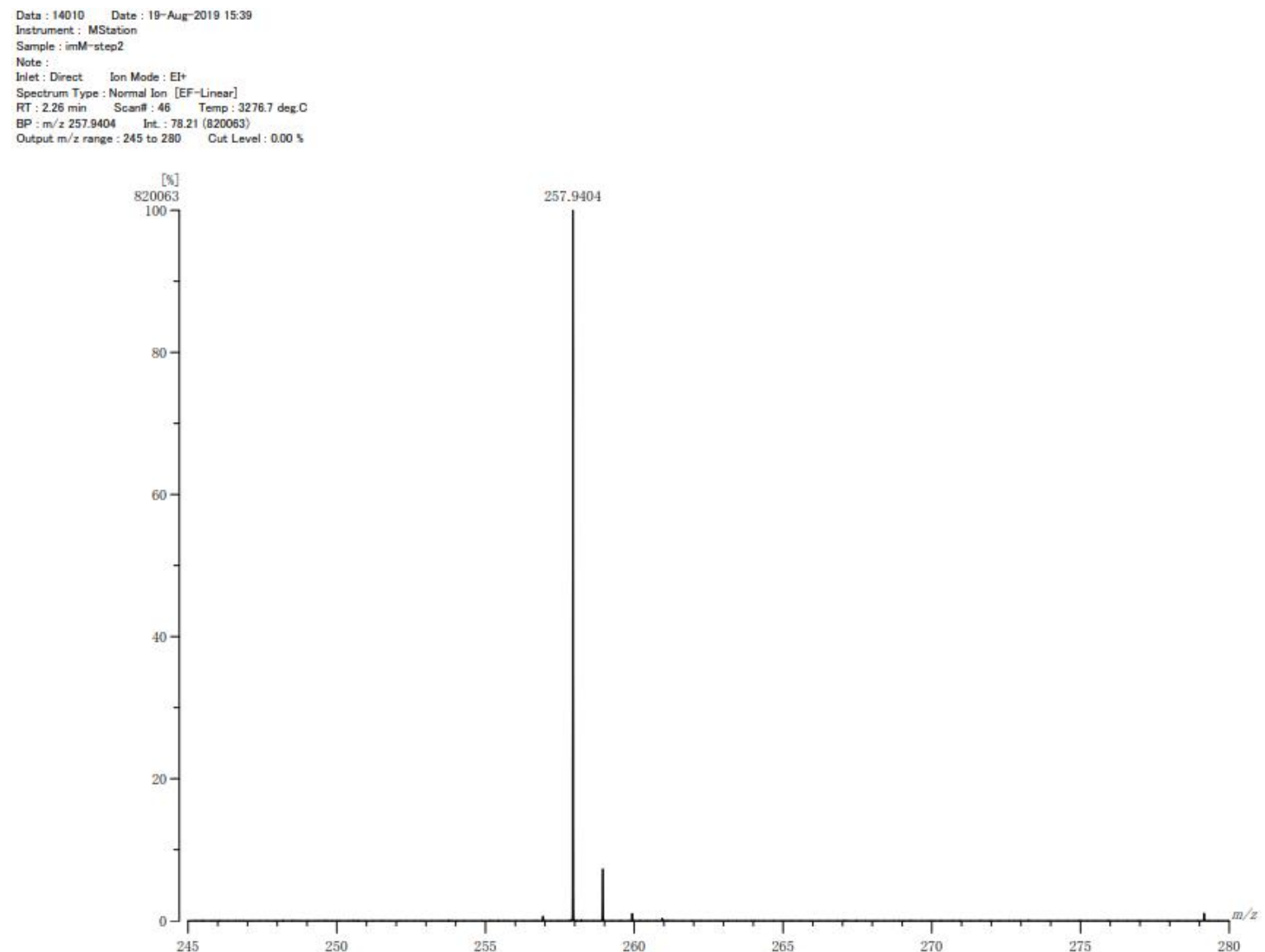

Figure S16. HRMS-EI ${ }^{+}$spectrum of compound 2. 


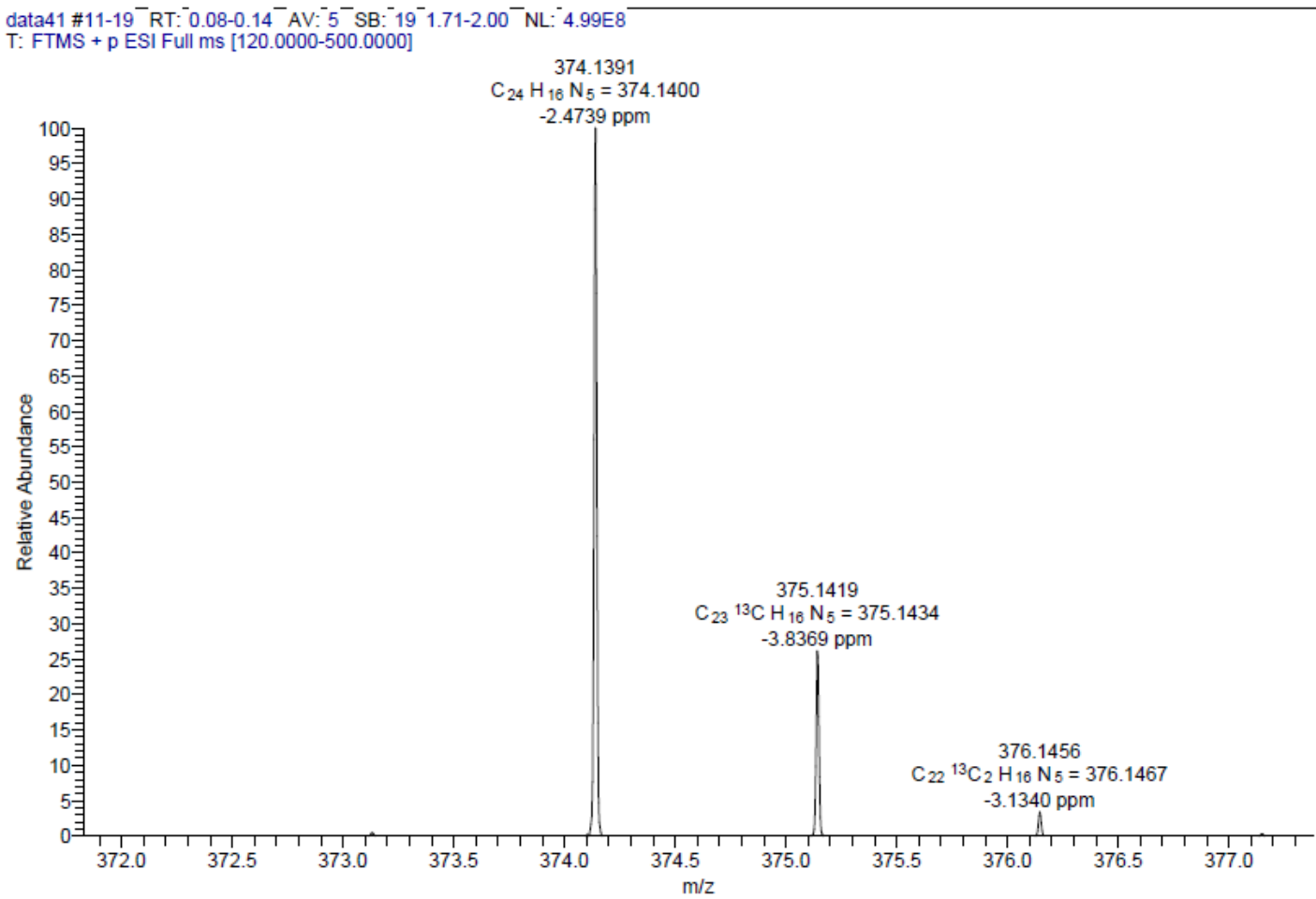

Figure S17. FTMS-ESI ${ }^{+}$spectrum of imM-o-Cz.

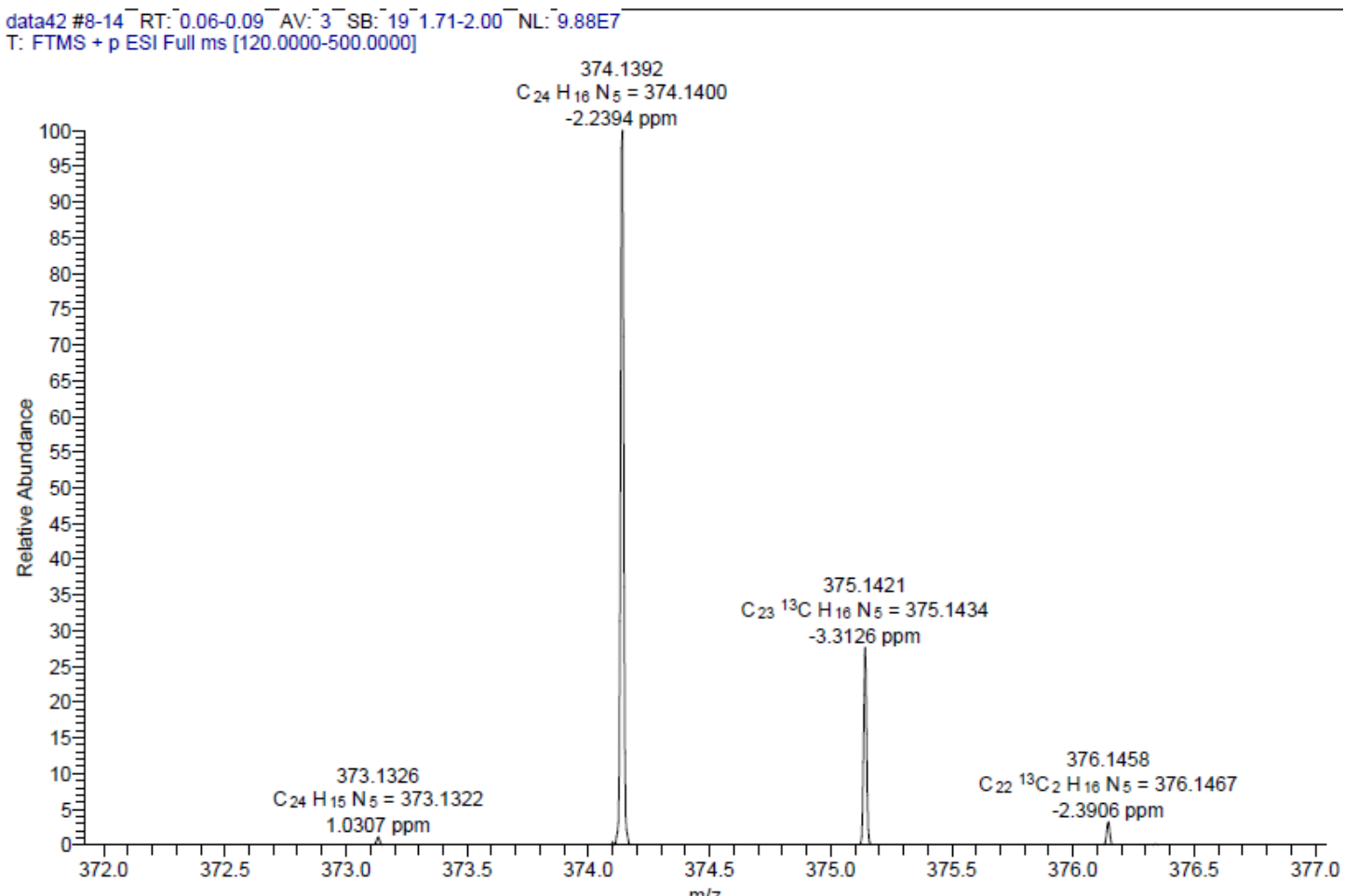

Figure S18. FTMS-ESI ${ }^{+}$spectrum of imM-m-Cz. 
data43 \#7-17 ${ }^{-} \mathrm{RT}^{-}{ }^{-} 0.05-0.12^{-} \mathrm{AV}^{-}{ }^{-}{ }^{-} \mathrm{SB}^{-}{ }^{-1} 19^{-} 1.71-2.00^{-} \mathrm{NL}:{ }^{-} 2.94 \mathrm{E} 7$ T: FTMS + p ESI Full ms [120.0000-500.0000]

$$
374.1390
$$

$\mathrm{C}_{24} \mathrm{H}_{16} \mathrm{~N}_{5}=374.1400$

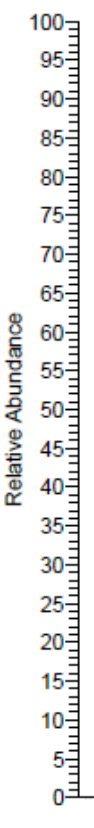

$-2.8051 \mathrm{ppm}$

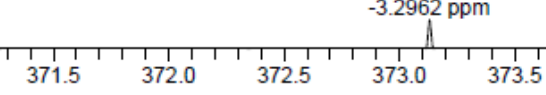

375.1420

$\mathrm{C}_{23}{ }^{13} \mathrm{CH}_{16} \mathrm{~N}_{5}=375.1434$

$-3.5582 \mathrm{ppm}$

Figure S19. FTMS-ESI ${ }^{+}$spectrum of imM-p-Cz. 
(a)

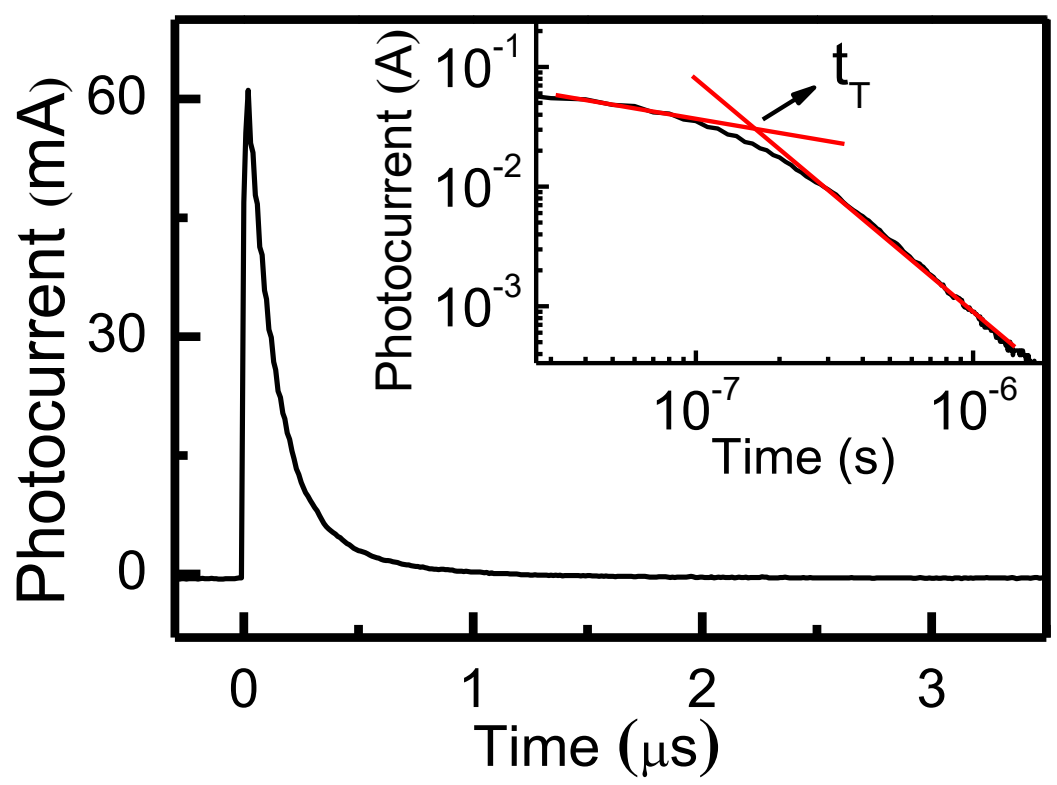

(b)

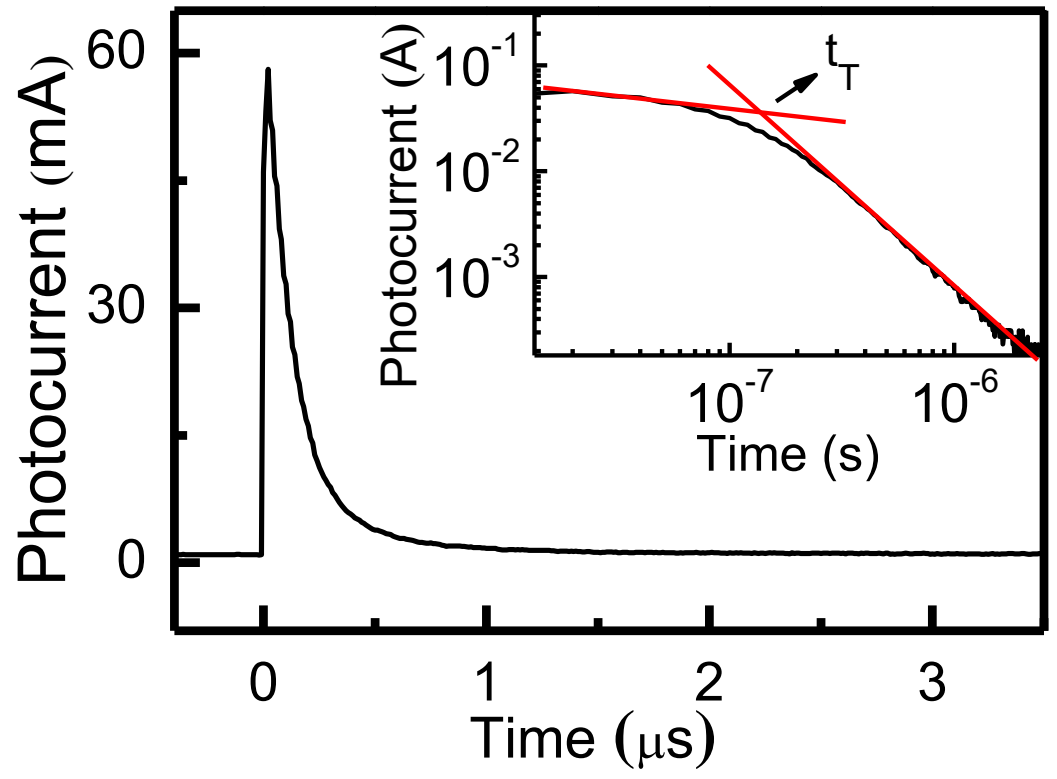


(c)

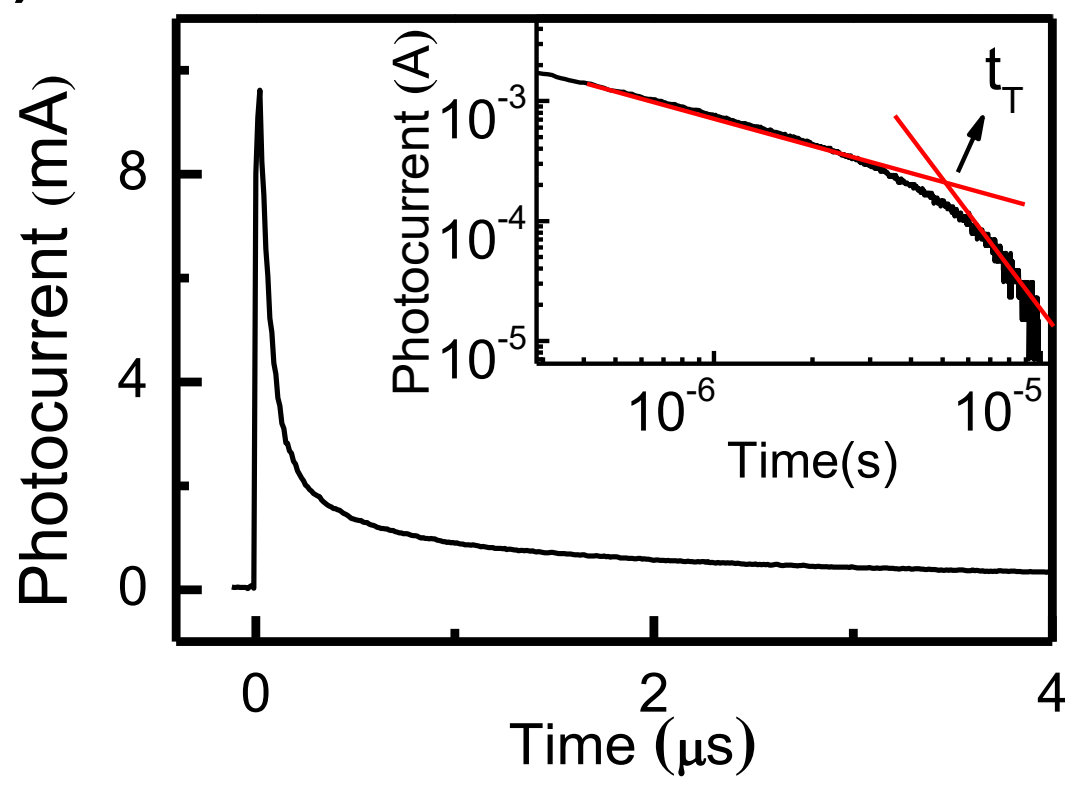

(d)

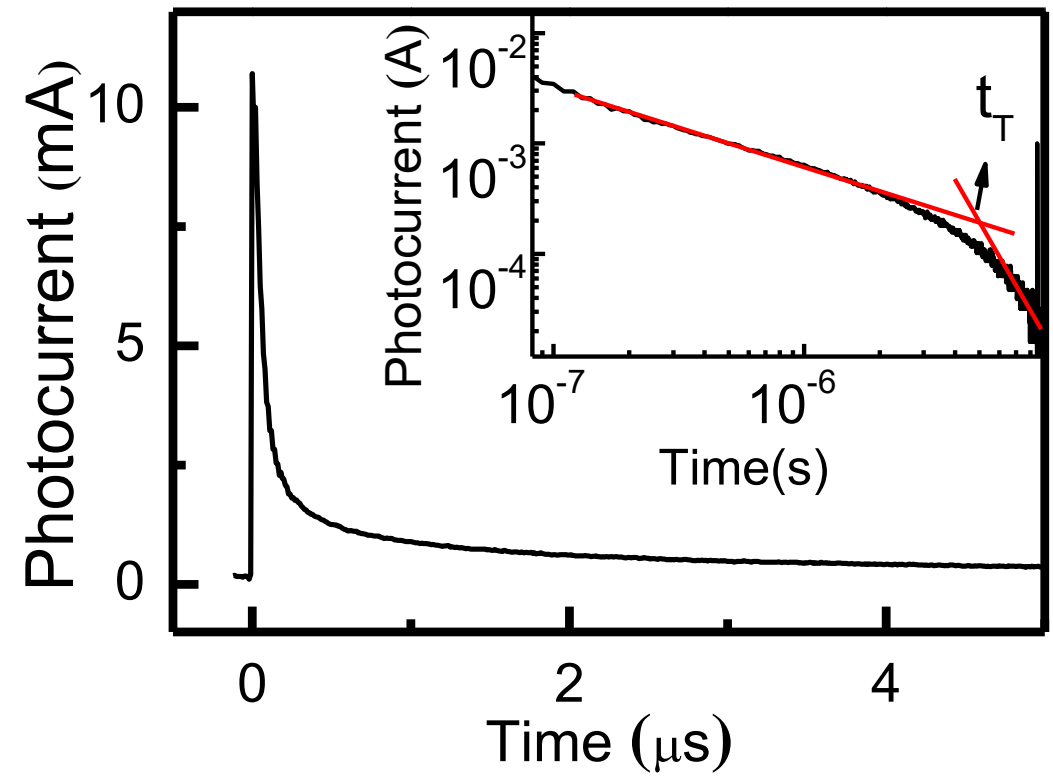


(e)

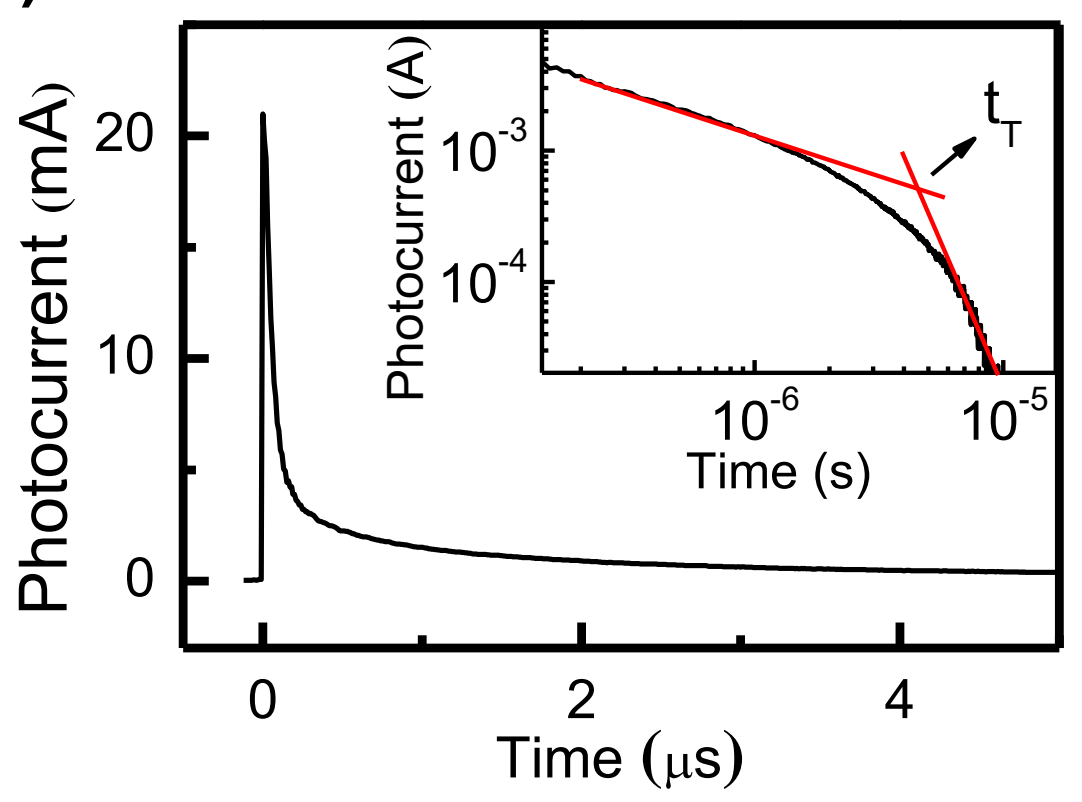

(f)

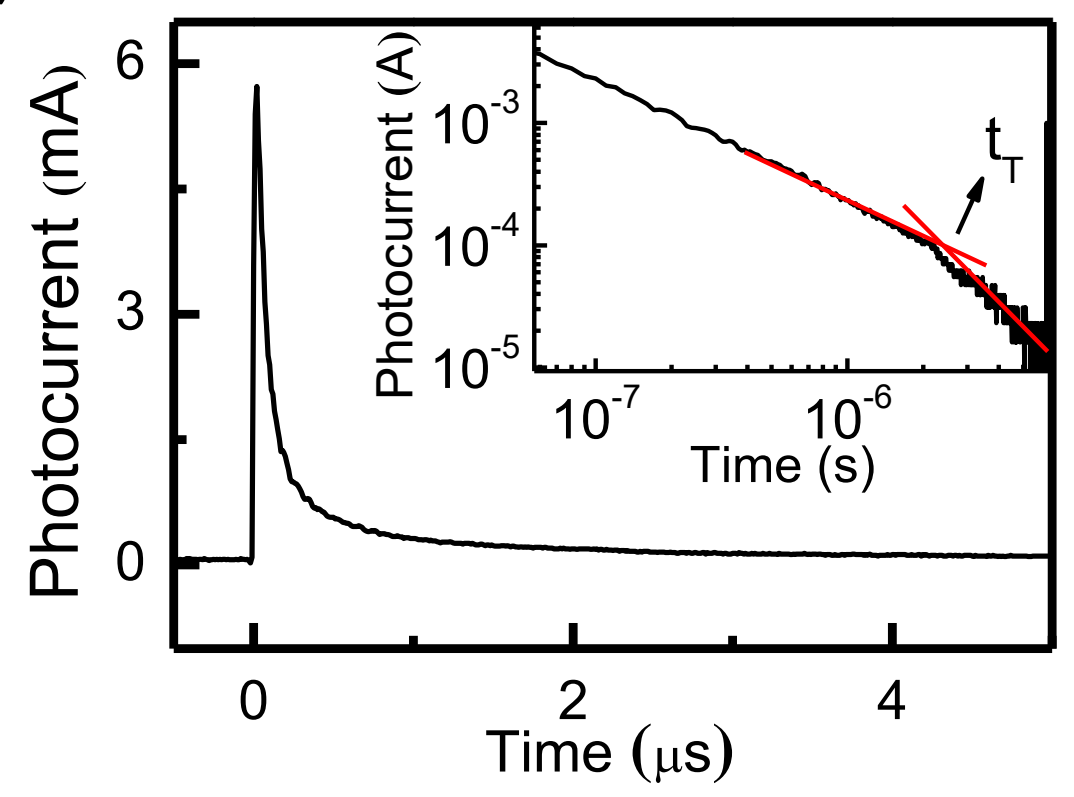

Figure 20. Representative TOF transient for electrons and holes of the compounds. (a) the hole of imM-o-Cz, (b) the electron of imM-o-Cz, (c) the hole of imM-m-Cz, (d) the electron of imM-m-Cz, (e) the hole of imM-p-Cz, (f) the electron of imM-p-Cz. 
Table S3. EL characteristics (with errors) of green PhOLEDs with different host materials.

\begin{tabular}{|c|c|c|c|c|c|c|c|c|}
\hline \multicolumn{2}{|l|}{ Device } & A1 & $\mathrm{A} 2$ & A3 & B & $\mathrm{C}$ & $\mathrm{D}$ & $\mathrm{CT}$ \\
\hline \multicolumn{2}{|l|}{ Host } & CBP & $\mathrm{mCP}$ & \multicolumn{5}{|c|}{ 26DCzPPy imM-p-Cz imM-m-Cz imM-o-Cz imM-m-Cz } \\
\hline \multirow{8}{*}{$\begin{array}{c}\text { External } \\
\text { Quantum } \\
\text { Efficiency (\%) }\end{array}$} & \multirow{2}{*}{$a$} & 21.2 & 15.0 & 15.2 & 19.5 & 21.9 & 18.7 & 40.6 \\
\hline & & $( \pm 0.7)$ & $( \pm 0.9)$ & $( \pm 1.8)$ & $( \pm 0.2)$ & $( \pm 1.1)$ & $( \pm 0.2)$ & $( \pm 0.6)$ \\
\hline & \multirow{3}{*}{$b$} & 16.7 & 9.8 & 12.7 & 16.3 & 18.2 & 15.4 & 35.6 \\
\hline & & $( \pm 0.2)$ & $( \pm 0.6)$ & $( \pm 1.4)$ & $( \pm 0.1)$ & $( \pm 0.1)$ & $( \pm 0.1)$ & $( \pm 0.6)$ \\
\hline & & 9.9 & 5.1 & 10.2 & 12.5 & 14.6 & 11.5 & 28.9 \\
\hline & \multirow{2}{*}{$c$} & $( \pm 0.5)$ & $( \pm 0.4)$ & $( \pm 1.0)$ & $( \pm 0.2)$ & $( \pm 0.1)$ & $( \pm 0.1)$ & $( \pm 0.5)$ \\
\hline & & 3.6 & & 5.4 & 6.8 & 7.5 & 5.2 & 18.4 \\
\hline & $d$ & $( \pm 0.3)$ & - & $( \pm 0.4)$ & $( \pm 0.3)$ & $( \pm 0.3)$ & $( \pm 0.1)$ & $( \pm 0.2)$ \\
\hline \multirow{8}{*}{$\begin{array}{l}\text { Luminance } \\
\text { Efficiency } \\
\left(\mathrm{cd} \mathrm{A}{ }^{-1}\right)\end{array}$} & \multirow[b]{2}{*}{$a$} & 75.9 & 52.8 & 53.3 & 69.3 & 77.9 & 65.7 & 151.2 \\
\hline & & $( \pm 2.5)$ & $( \pm 3.3)$ & $( \pm 6.2)$ & $( \pm 0.6)$ & $( \pm 4.0)$ & $( \pm 0.8)$ & $( \pm 2.1)$ \\
\hline & \multirow{2}{*}{$b$} & 59.7 & 34.6 & 44.6 & 57.8 & 64.7 & 54.3 & 132.6 \\
\hline & & $( \pm 0.5)$ & $( \pm 2.1)$ & $( \pm 5.0)$ & $( \pm 0.5)$ & $( \pm 0.1)$ & $( \pm 0.1)$ & $( \pm 2.1)$ \\
\hline & \multirow{3}{*}{$c$} & 35.4 & 12.8 & 35.7 & 44.3 & 52.1 & 40.6 & 107.7 \\
\hline & & $( \pm 1.9)$ & $( \pm 1.5)$ & $( \pm 3.5)$ & $( \pm 0.7)$ & $( \pm 0.1)$ & $( \pm 0.3)$ & $( \pm 1.7)$ \\
\hline & & 13.1 & & 19.0 & 24.0 & 26.8 & 18.4 & 68.8 \\
\hline & $d$ & $( \pm 1.2)$ & - & $( \pm 1.4)$ & $( \pm 1.1)$ & $( \pm 1.1)$ & $( \pm 0.1)$ & $( \pm 0.1)$ \\
\hline \multirow{8}{*}{ 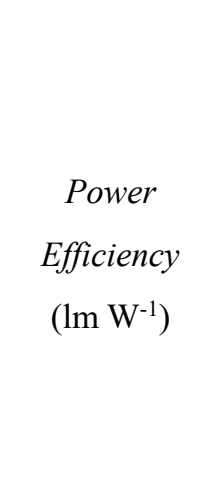 } & \multirow{2}{*}{$a$} & 91.8 & 64.2 & 45.6 & 90.8 & 102.1 & 72.8 & 98.6 \\
\hline & & $( \pm 3.0)$ & $( \pm 2.3)$ & $( \pm 4.8)$ & $( \pm 0.8)$ & $( \pm 5.3)$ & $( \pm 2.0)$ & $( \pm 0.7)$ \\
\hline & \multirow[b]{2}{*}{$b$} & 42.5 & 21.3 & 25.9 & 52.1 & 59.3 & 38.4 & 61.2 \\
\hline & & $( \pm 0.4)$ & $( \pm 1.3)$ & $( \pm 2.9)$ & $( \pm 0.5)$ & $( \pm 0.1)$ & $( \pm 0.1)$ & $( \pm 1.0)$ \\
\hline & \multirow{2}{*}{$c$} & 15.1 & 6.7 & 15.5 & 26.8 & 31.3 & 19.8 & 31.8 \\
\hline & & $( \pm 0.8)$ & $( \pm 0.6)$ & $( \pm 1.5)$ & $( \pm 0.4)$ & $( \pm 0.1)$ & $( \pm 0.1)$ & $( \pm 0.5)$ \\
\hline & \multirow[b]{2}{*}{$d$} & 3.3 & & 5.6 & 7.8 & 8.9 & 5.4 & 12.1 \\
\hline & & $( \pm 0.3)$ & - & $( \pm 0.4)$ & $( \pm 0.4)$ & $( \pm 0.4)$ & $( \pm 0.1)$ & $( \pm 0.1)$ \\
\hline \multirow[b]{2}{*}{$V_{o n}(\mathrm{~V})$} & \multirow[b]{2}{*}{$e$} & 2.3 & 2.5 & 3.6 & 2.3 & 2.3 & 2.7 & 4.6 \\
\hline & & $( \pm 0.1)$ & $( \pm 0.1)$ & $( \pm 0.4)$ & $( \pm 0.1)$ & $( \pm 0.1)$ & $( \pm 0.1)$ & $( \pm 0.1)$ \\
\hline \multirow{3}{*}{\multicolumn{2}{|c|}{$\begin{array}{c}\text { Max Luminance } \\
\left(\mathrm{cd} \mathrm{m}^{-2}\right)\end{array}$}} & 1.40 & 8.09 & 1.88 & 1.59 & 1.68 & 1.39 & 2.80 \\
\hline & & $( \pm 0.08)$ & $( \pm 1.72)$ & $( \pm 0.34)$ & $( \pm 0.01)$ & $( \pm 0.20)$ & $( \pm 0.07)$ & $( \pm 0.12)$ \\
\hline & & $\times 10^{5}$ & $\times 10^{4}$ & $\times 10^{5}$ & $\times 10^{5}$ & $\times 10^{5}$ & $\times 10^{5}$ & $\times 10^{5}$ \\
\hline
\end{tabular}

